\title{
RATIONAL SMOOTHNESS OF VARIETIES OF REPRESENTATIONS FOR QUIVERS OF DYNKIN TYPE
}

\author{
PHILIPPE CALDERO AND RALF SCHIFFLER
}

\begin{abstract}
Let $\mathbf{U}^{+}$be the positive part of the quantized enveloping algebra $\mathbf{U}$ of type $A, D$ or $E$. The change of basis between canonical, and PBW-basis of $\mathbf{U}^{+}$has a geometric interpretation in terms of local intersection cohomology of some affine algebraic varieties, namely the Zariski closures of orbits of representations of a quiver of type $A, D$ or $E$. In this paper we characterize the rationally smooth orbit closures and prove in particular that orbit closures are smooth if and only if they are rationally smooth. This provides an analogue of theorems of V. Deodhar, and J. Carrell and D. Peterson on Schubert varieties.
\end{abstract}

\section{INTRODUCTION}

Let $\mathbb{F}$ be an algebraically closed field, $\mathbf{d}=\left(d_{1}, d_{2}, \ldots, d_{n}\right) \in \mathbb{N}^{n}$ and $G_{\mathbf{d}}=$ $\prod_{i=1}^{n} G L_{d_{i}}(\mathbb{F})$. Let $\mathcal{Q}$ be a fixed quiver whose underlying graph $\Delta$ is the Dynkin graph of type $A_{n}, D_{n}$ or $E_{n} . G_{\mathbf{d}}$ acts on $E_{\mathbf{d}}=\oplus_{i \rightarrow j \in \mathcal{Q}} \operatorname{Hom}_{\mathbb{F}}\left(\mathbb{F}^{d_{i}}, \mathbb{F}^{d_{j}}\right)$, by conjugation. Let $\mathcal{O}$ be a $G_{\mathrm{d}}$-orbit and $\overline{\mathcal{O}}$ its Zariski closure. In $[\mathrm{BS}$ the complete list of rationally smooth orbit closures of type $A_{n}$ was obtained. As a consequence it was shown that, in type $A_{n}, \overline{\mathcal{O}}$ is rationally smooth if and only if $\overline{\mathcal{O}}$ is smooth. In this paper we will generalize these results to the types $D_{n}$ and $E_{n}$, see theorem 3.6] and corollary 3.7

Rational smoothness is a topological property, which is defined using local intersection cohomology, and has been extensively studied for Schubert varieties, Deo85, Car94. For a survey of some of these results, see BL00 and Bri98.

Let $\mathbf{U}^{+}$be the positive part of the quantized enveloping algebra $\mathbf{U}$ over $\mathbb{Q}(v)$ associated by Drinfeld and Jimbo to the root system of type $\Delta$. Kashiwara and Lusztig have constructed independently of each other a unique canonical basis $\mathbf{B}$ of $\mathbf{U}^{+}$in Kas91 and Lus90a. For each reduced expression $\mathbf{i}$ of the longest element $w_{0}$ of the Weyl group $W$ of type $\Delta$, there is also a PBW-basis $B_{\mathbf{i}}$. Some of the reduced expressions are adapted to the quiver $\mathcal{Q}$. In this case, Lusztig has shown in Lus90a that the entries of the transition matrix between the bases $\mathbf{B}$ and $B_{\mathbf{i}}$ have a description in terms of local intersection cohomology of orbit closures. We use this approach to study rational smoothness of orbit closures. One important ingredient therefore is the action of the bar involution of $\mathbf{U}$ on the elements of the PBW-basis.

This paper is organized as follows. In section 2 we fix notations and recall some results that we will need at a later stage. In particular, we recall the Hall algebra realization of $\mathbf{U}^{+}$. We then present two different approaches to study rational smoothness of orbit closures: one algebraic and the other geometric.

2000 Mathematics Subject Classification. Primary 17B37.

The second author was supported in part by FCAR Grant. 
Section 3 contains the algebraic approach. Here we consider the bar of a PBWbasis element as a linear combination in the PBW-basis, and study the coefficients of this expansion; to be more precise, we calculate the derivative at $v=1$ of these coefficients. The method used to calculate the coefficients differs from BS. In fact, we use the dual approach, with the help of the canonical form on $\mathbf{U}^{+}$. Indeed, the dual PBW-basis is known in the Hall algebra context by Gre95. Moreover, the adjoint $\sigma$ of the bar involution is a $\mathbb{Q}$-antiautomorphism (up to a power of $q$ ) given by Lusztig, Lus93. The reason why things are more convenient when we dualize is that the dual of an irreducible element of the PBW-basis is an element of the dual canonical basis, Cal, and so is stable by $\sigma$. Then, the image by $\sigma$ of a general dual PBW-basis element can be easily calculated by the antiautomorphism property. This enables us to realize the desired coefficients in terms of generalized Hall polynomials, see Proposition 3.1] Using the same methods as in BS], we then obtain the complete list of rationally smooth orbit closures. It is easy to see that each orbit closure in this list is smooth.

In section 4 we present another proof of this characterization using a geometric approach. According to an idea of Michel Brion, we calculate the Euler-Poincaré characteristic of the projectivization of the orbit closures $\overline{\mathcal{O}}$. Indeed, it is known that, for rationally smooth cones, the Euler-Poincaré characteristic of the projectivization of the cone equals the dimension of the cone. Thanks to a theorem of Deligne on the Weil conjecture, the characteristic can be calculated by counting the number of $\mathbb{F}_{q}$-rational points of an orbit closure viewed as a variety on an algebraic closure $\overline{\mathbb{F}_{q}}$ of $\mathbb{F}_{q}$, and specializing at $q=1$. This method provides a geometric interpretation of the proof given in $[\mathrm{BS}$, and followed in 3. It also provides an interesting homological realization of algebraic elements, namely the derivative at $q=1$ of the coefficients of the bar involution in the PBW-basis.

Our main result, Theorem 3.3 generalizes [BS, Theorem 5.4]. Nevertheless, the E-case slightly differs from the A-D case. Let's explain how. First of all, by Ringel's Hall algebra approach of quantum groups, the coefficients of the bar automorphism discussed above are parameterized by couples $\left(\mathcal{O}, \mathcal{O}^{\prime}\right)$ of $G_{\mathbf{d}}$ orbits, and are non zero if and only if $\mathcal{O}^{\prime} \preceq \mathcal{O}$ for the so-called degeneration ordering. Now, Klaus Bongartz gave, Bon95, a description of this ordering in the representation theory of the quiver $\mathcal{Q}$. In particular, we can define "elementary degenerations" corresponding to certain non-split exact sequences, called elementary operations, in the AuslanderReiten quiver of $\mathcal{Q}$, and $\mathcal{O}^{\prime} \preceq \mathcal{O}$ if and only if there exists a chain of elementary degenerations relying $\mathcal{O}^{\prime}$ and $\mathcal{O}$. The results in [BS] proves that the coefficient parameterized by $\left(\mathcal{O}, \mathcal{O}^{\prime}\right)$ "sees" the minimal length of such a chain, and that the derivative at $q=1$ of the coefficient is non zero if and only if the degeneration corresponding to $\left(\mathcal{O}, \mathcal{O}^{\prime}\right)$ is elementary. In order to generalize this result, we have to replace elementary degenerations by a finer notion, see 3.2.

\section{Notations AND RECOLLECTIONS}

2.1. The quantized enveloping algebra $\mathbf{U}$. Let $\Delta$ be a Dynkin diagram of type $A, D$ or $E$, let $n$ be the number of vertices of $\Delta$ and let $\left(a_{i j}\right)$ be the corresponding Cartan matrix. Thus

$$
a_{i j}= \begin{cases}2 & \text { if } i=j \\ -1 & \text { if } i-j \text { is an edge in } \Delta \\ 0 & \text { otherwise. }\end{cases}
$$


Let $v$ be an indeterminate and $\mathbf{U}$ the quantized enveloping algebra of DrinfeldJimbo of type $\Delta$ over the field $\mathbb{Q}(v)$ of rational functions. $\mathbf{U}$ is a $\mathbb{Q}(v)$-algebra with generators: $E_{i}, F_{i}, K_{i}, K_{i}^{-1}(1 \leq i \leq n)$ and relations:

$$
\begin{gathered}
K_{i} K_{i}^{-1}=K_{i}^{-1} K_{i}=1, \quad K_{i} K_{j}=K_{j} K_{i} ; \\
K_{i} E_{j}=v^{a_{i j}} E_{j} K_{i} \\
K_{i} F_{j}=v^{-a_{i j}} \\
E_{i} F_{j}-F_{j} E_{i}=\delta_{i j} \frac{\left(K_{i}-K_{i}^{-1}\right)}{\left(v-v^{-1}\right)} \quad \text { where } \delta_{i j}= \begin{cases}1 & \text { if } i=j \\
0 & \text { if } i \neq j .\end{cases} \\
\begin{cases}E_{i}^{2} E_{j}-\left(v+v^{-1}\right) E_{i} E_{j} E_{i}+E_{j} E_{i}^{2}=0 & \text { if } a_{i j}=-1 \\
E_{i} E_{j}-E_{j} E_{i}=0 & \text { if } a_{i j} \neq-1 .\end{cases} \\
\begin{cases}F_{i}^{2} F_{j}-\left(v+v^{-1}\right) F_{i} F_{j} F_{i}+F_{j} F_{i}^{2}=0 & \text { if } a_{i j}=-1 \\
F_{i} F_{j}-F_{j} F_{i}=0, & \text { if } a_{i j} \neq-1\end{cases}
\end{gathered}
$$

Let $\mathbf{U}^{+}$be the $\mathbb{Q}(v)$-subalgebra generated by the $E_{i} \quad(1 \leq i \leq n)$. Let $\overline{()}$ : $\mathbf{U} \rightarrow \mathbf{U}$ be the involution of $\mathbb{Q}$-algebras defined by

$$
E_{i} \mapsto E_{i}, \quad F_{i} \mapsto F_{i}, \quad K_{i} \mapsto K_{i}^{-1} \quad \text { for all } 1 \leq i \leq n \quad \text { and } \quad v \mapsto v^{-1} .
$$

Note that $\overline{\mathbf{U}^{+}}=\mathbf{U}^{+}$.

Let $Q$, resp. $Q^{+}$, be the free abelian group, resp. semigroup, with basis $\left\{\alpha_{1}, \alpha_{2}, \ldots, \alpha_{n}\right\}$. Define an inner product $(,)_{Q}$ on $Q$ by $\left(\alpha_{i}, \alpha_{j}\right)_{Q}=a_{i j}$. Let $R=\left\{\alpha \in Q \mid(\alpha, \alpha)_{Q}=2\right\} . R$ is a root system of type $\Delta$ whose set of simple roots is $\left\{\alpha_{1}, \alpha_{2}, \ldots, \alpha_{n}\right\}$. Let $R^{+}=\left\{\alpha \in R \mid \alpha=\sum_{j} c_{j} \alpha_{j}\right.$ with $\left.c_{j} \in \mathbb{N}\right\}$ be the subset of positive roots.

Each $\alpha \in R$ defines a reflection $s_{\alpha}: Q \rightarrow Q, z \mapsto z-(z, \alpha)_{Q} \alpha$. We will write $s_{i}$ instead of $s_{\alpha_{i}}$. Let $W$ be the Weyl group of $R$. This is the subgroup of $\operatorname{Aut}(Q)$ generated by the reflections $s_{i},(1 \leq i \leq n)$. Let $\ell(w)$ be the length of $w$ with respect to the generators $\left\{s_{1}, s_{2}, \ldots, s_{n}\right\}$ and denote by $w_{0}$ the unique element of $W$ of maximal length. It is known that $\ell\left(w_{0}\right)=\nu=\#\left(R^{+}\right)$. Let Tr be the linear form on $Q^{+}$such that $\operatorname{Tr}\left(\alpha_{i}\right)=1,1 \leq i \leq n$.

We shall use the $Q^{+}$-grading wt of $\mathbf{U}^{+}$defined by $\operatorname{wt}\left(E_{i}\right)=\alpha_{i}$.

Lusztig has defined an action of the braid group on $\mathbf{U}$ [Lus90a and used it to define bases of PBW type of $\mathbf{U}^{+}$. We now recall these definitions.

For $i \in\{1, \ldots, n\}$, let $\tilde{T}_{i}: \mathbf{U} \rightarrow \mathbf{U}$ be the automorphism of $\mathbb{Q}(v)$-algebras defined by

$$
\begin{array}{llll}
E_{i} \mapsto-K_{i}^{-1} F_{i}, & F_{i} \mapsto-E_{i} K_{i}, & K_{i} \mapsto K_{i}^{-1}, & \\
E_{j} \mapsto E_{j}, & F_{j} \mapsto F_{j}, & K_{j} \mapsto K_{j}, & \text { if } a_{i j}=0, \\
E_{j} \mapsto\left(E_{j} E_{i}-v^{-1} E_{i} E_{j}\right), & F_{j} \mapsto\left(F_{i} F_{j}-v F_{j} F_{i}\right), & K_{j} \mapsto K_{i} K_{j}, & \text { if } a_{i j}=-1 .
\end{array}
$$

We have $\tilde{T}_{i} \tilde{T}_{j} \tilde{T}_{i}=\tilde{T}_{j} \tilde{T}_{i} \tilde{T}_{j}$ if $a_{i j}=-1$ and $\tilde{T}_{i} \tilde{T}_{j}=\tilde{T}_{j} \tilde{T}_{i} \quad$ if $a_{i j} \neq-1$. This gives us a braid group action. Moreover $\tilde{T}_{i}\left(E_{j}\right)=\tilde{T}_{j}^{-1}\left(E_{i}\right)$ if $a_{i j}=-1$.

Given integers $M, N \geq 0$, we define

$$
[N] !=\prod_{h=1}^{N} \frac{\left(v^{h}-v^{-h}\right)}{\left(v-v^{-1}\right)} \in \mathbb{Z}\left[v, v^{-1}\right],\left[\begin{array}{c}
M+N \\
N
\end{array}\right]=\frac{[M+N] !}{[M] ![N] !} \in \mathbb{Z}\left[v, v^{-1}\right]
$$




$$
\text { and } \quad E_{i}^{(N)}=\frac{E_{i}^{N}}{[N] !} \text { for } 1 \leq i \leq n .
$$

Let $\mathcal{I}$ be the set of sequences $\mathbf{i}=\left(i_{1}, \ldots, i_{\nu}\right)$ of elements in $\{1, \ldots, n\}$ such that $s_{i_{1}} \ldots s_{i_{\nu}}$ is a reduced expression of $w_{0}$. Each $\mathbf{i} \in \mathcal{I}$ gives rise to a total order on $R^{+}=\left\{\alpha^{1}, \ldots, \alpha^{\nu}\right\}$, where $\alpha^{t}=s_{i_{1}} s_{i_{2}} \cdots s_{i_{t-1}}\left(\alpha_{i_{t}}\right)$ for $t=1, \ldots, \nu$. We say that an element $\mathbf{c}=\left(c_{1}, \ldots, c_{\nu}\right) \in \mathbb{N}^{\nu}$ is of i-homogeneity $\mathbf{d}=\left(d_{1}, \ldots, d_{n}\right) \in \mathbb{N}^{n}$ if

$$
\sum_{t=1}^{\nu} c_{t} \alpha^{t}=\sum_{k=1}^{n} d_{k} \alpha_{k}
$$

Let $\mathbf{b}(t)$ be the vector $(0, \ldots, 0,1,0, \ldots, 0) \in \mathbb{N}^{\nu}$ whose only nonzero component is in the $t^{\text {th }}$ column and is 1 , where $1 \leq t \leq \nu$.

For $\mathbf{i}=\left(i_{1}, \ldots, i_{\nu}\right) \in \mathcal{I}$ and $\mathbf{c}=\left(c_{1}, \ldots, c_{\nu}\right) \in \mathbb{N}^{\nu}$, define

$$
E_{\mathbf{i}}^{\mathbf{c}}=E_{i_{1}}^{\left(c_{1}\right)} \tilde{T}_{i_{1}}\left(E_{i_{2}}^{\left(c_{2}\right)}\right) \tilde{T}_{i_{1}} \tilde{T}_{i_{2}}\left(E_{i_{3}}^{\left(c_{3}\right)}\right) \ldots \tilde{T}_{i_{1}} \tilde{T}_{i_{2}} \ldots \tilde{T}_{i_{(\nu-1)}}\left(E_{i_{\nu}}^{\left(c_{\nu}\right)}\right)=\prod_{t=1}^{\nu} E_{\mathbf{i}}^{c_{\mathbf{t}} \mathbf{b}(t)} .
$$

Note that if $\mathbf{c}$ is of $\mathbf{i}$-homogeneity $\mathbf{d}$ then $\operatorname{Tr}\left(\operatorname{wt}\left(E_{\mathbf{i}}^{\mathbf{c}}\right)\right)=\sum_{k=1}^{n} d_{k}$

Proposition 2.1. Let $\mathbf{i} \in \mathcal{I}$. Then $B_{\mathbf{i}}=\left\{E_{\mathbf{i}}^{\mathbf{c}} \mid \mathbf{c} \in \mathbb{N}^{\nu}\right\}$ is a $\mathbb{Q}(v)$-basis of $\mathbf{U}^{+}$. We say that $B_{\mathbf{i}}$ is a basis of $P B W$ type.

Proof. Lus90b, sect. 1.8 and 1.13]

We now recall Lusztig's construction of the canonical basis of $\mathbf{U}^{+}$.

Theorem 2.2. Let $\mathbf{i} \in \mathcal{I}$ and $\mathcal{L}_{\mathbf{i}}$ the $\mathbb{Z}\left[v^{-1}\right]$-submodule of $\mathbf{U}^{+}$generated by $B_{\mathbf{i}}$.

(i) $\mathcal{L}_{\mathbf{i}}$ is independent of $\mathbf{i}$. We denote $\mathcal{L}_{\mathbf{i}}$ by $\mathcal{L}$.

(ii) $\pi\left(B_{\mathbf{i}}\right)$ is a $\mathbb{Z}$-basis of $\mathcal{L} / v^{-1} \mathcal{L}$ independent of $\mathbf{i}$. Here $\pi: \mathcal{L} \rightarrow \mathcal{L} / v^{-1} \mathcal{L}$ is the canonical projection. We denote $\pi\left(B_{\mathbf{i}}\right)$ by $B$.

(iii) The restriction of $\pi: \mathcal{L} \rightarrow \mathcal{L} / v^{-1} \mathcal{L}$ defines an isomorphism of $\mathbb{Z}$-modules $\pi^{\prime}: \mathcal{L} \cap \overline{\mathcal{L}} \rightarrow \mathcal{L} / v^{-1} \mathcal{L}$ where $\overline{\mathcal{L}}$ is the image of $\mathcal{L}$ under $\overline{(~)}$. In particular, $\mathbf{B}=\pi^{\prime-1}(B)$ is a $\mathbb{Z}$-basis of $\mathcal{L} \cap \overline{\mathcal{L}}$.

(iv) $\mathbf{B}$ is a $\mathbb{Z}\left[v^{-1}\right]$-basis of $\mathcal{L}$ and $a \mathbb{Q}(v)$-basis of $\mathbf{U}^{+} . \mathbf{B}$ is said to be the canonical basis of $\mathbf{U}^{+}$.

(v) Each element of $\mathbf{B}$ is fixed by $\overline{()}: \mathbf{U}^{+} \rightarrow \mathbf{U}^{+}$.

Proof. Lus90a

2.2. Specialization at $v=1$. Let $\mathcal{A}=\mathbb{Q}[v]_{(v-1)}$ denote the subring of $\mathbb{Q}(v)$ consisting of functions regular at $v=1$. Define the $\mathcal{A}$-form $\mathbf{U}_{\mathcal{A}}$ of $\mathbf{U}^{+}$to be the $\mathcal{A}$-subalgebra of $\mathbf{U}^{+}$generated by $E_{i}, 1 \leq i \leq n$. Denote by $\mathbf{U}_{1}^{+}=\mathbf{U}_{\mathcal{A}} /(v-$ 1) $\mathbf{U}_{\mathcal{A}}$ the specialization of $\mathbf{U}^{+}$at $v=1$. This is the positive part of the classical universal enveloping algebra of type $\Delta$, with generators $E_{1}, \ldots, E_{n}$ and classical Serre relations

$$
\begin{cases}E_{i}^{2} E_{j}-2 E_{i} E_{j} E_{i}+E_{j} E_{i}^{2}=0 & \text { if } a_{i j}=-1 \\ E_{i} E_{j}-E_{j} E_{i}=0 & \text { if } a_{i j} \neq-1\end{cases}
$$

Note that in $\mathbf{U}_{1}, \tilde{T}_{i}\left(E_{j}\right)$ becomes the usual bracket $E_{j} E_{i}-E_{i} E_{j}$ and the specialization $B_{\mathbf{i}}(1)=\left\{E_{\mathbf{i}}^{\mathbf{c}}(1) \mid \mathbf{c} \in \mathbb{N}^{\nu}\right\}$ of the PBW-basis is a PBW-basis of $\mathbf{U}_{1}$. 
2.3. Quiver modules. Let $\mathcal{Q}=\left(\mathcal{Q}^{0}, \mathcal{Q}^{1}\right)$ be a quiver whose underlying graph is $\Delta$, i.e. for each edge $\{i, j\}$ of $\Delta$ we fix an orientation. (We use the notation $\mathcal{Q}^{0}$ for the set of vertices of the quiver $\mathcal{Q}$ and $\mathcal{Q}^{1}$ for the set of arrows.) A vertex $i \in \mathcal{Q}^{0}$ is a sink (respectively a source) of $\mathcal{Q}$ if there is no arrow $i \rightarrow j$ (respectively $i \leftarrow j$ ) $\in \mathcal{Q}^{1}$. An element $\mathbf{i}=\left(i_{1}, \ldots, i_{\nu}\right) \in \mathcal{I}$ is adapted to the quiver $\mathcal{Q}$ if $i_{1}$ is a sink of $\mathcal{Q}_{1}=\mathcal{Q}$ and $i_{k}$ is a sink of the quiver $\mathcal{Q}_{k}=s_{i_{k-1}}\left(\mathcal{Q}_{k-1}\right)$ obtained from $\mathcal{Q}_{k-1}$ by reversing the orientation of all arrows ending at $i_{k-1}$, where $2 \leq k \leq \nu$. It is easy to see that there is an element $\mathbf{i} \in \mathcal{I}$ adapted to $\mathcal{Q}$ and that an element $\mathbf{i}$ of $\mathcal{I}$ can be adapted to at most one quiver.

For the rest of this paper, let $\mathbf{i}$ be adapted to the quiver $\mathcal{Q}$.

Let $F$ be any field. A module (or representation) $\mathbf{V}=\left(V_{i}, f_{i j}\right)$ of $\mathcal{Q}$ is a collection of $n$ finite dimensional $F$-vector spaces $V_{i},(1 \leq i \leq n)$ and of $(n-1) F$-linear maps $f_{i j}: V_{i} \rightarrow V_{j},\left(i \rightarrow j \in \mathcal{Q}^{1}\right)$. A morphism from the module $\mathbf{V}=\left(V_{i}, f_{i j}\right)$ to the module $\mathbf{V}^{\prime}=\left(V_{i}^{\prime}, f_{i j}^{\prime}\right)$ is a collection of $F$-linear maps $g_{i}: V_{i} \rightarrow V_{i}^{\prime}, 1 \leq i \leq n$ such that $f_{i j}^{\prime} \circ g_{i}=g_{j} \circ f_{i j}$ for each $i \rightarrow j \in \mathcal{Q}^{1}$. These modules and morphisms form an abelian category $\operatorname{Mod}(\mathcal{Q})$. If $\mathbf{V}$ is a module of $\mathcal{Q}$, denote by $[\mathbf{V}]$ its isomorphism class in $\operatorname{Mod}(\mathcal{Q})$.

The dimension of the module $\mathbf{V}=\left(V_{i}, f_{i j}\right)$ is the $n$-tuple

$$
\operatorname{dim}(\mathbf{V})=\left(\operatorname{dim}_{F}\left(V_{1}\right), \operatorname{dim}_{F}\left(V_{2}\right), \ldots, \operatorname{dim}_{F}\left(V_{n}\right)\right) \in \mathbb{N}^{n} .
$$

A module $\mathbf{V}$ of $\mathcal{Q}$ is indecomposable if $\mathbf{V}$ cannot be written as the direct sum of proper submodules.

Theorem 2.3. $\quad$ (i) For all $\alpha \in R^{+}$, there is a unique indecomposable module (up to isomorphism), denoted $\mathbf{e}_{\alpha} \in \operatorname{Mod}(\mathcal{Q})$, such that $\operatorname{dim}\left(\mathbf{e}_{\alpha}\right)=$ $\left(d_{1}, \ldots, d_{n}\right)$ and $\alpha=\sum_{i=1}^{n} d_{i} \alpha_{i}$; any indecomposable module is isomorphic to $\mathbf{e}_{\alpha}$ for a unique $\alpha$. This is Gabriel's theorem.

(ii) There exists an ordering $\alpha^{t}, 1 \leq t \leq \nu$ of the positive roots such that $\operatorname{Hom}_{\mathcal{Q}}\left(\mathbf{e}_{\alpha^{t}}, \mathbf{e}_{\alpha^{s}}\right)=0$ if $s<t$.

(iii) There exists a bijection $\mathbf{c}=\left(c_{1}, c_{2}, \ldots, c_{\nu}\right) \mapsto[\mathbf{e}(\mathbf{c})]$ between $\mathbb{N}^{\nu}$ and the set of isomorphism classes of modules of $\mathcal{Q}$, where $\mathbf{e}(\mathbf{c})=\oplus_{t=1}^{\nu} c_{t} \mathbf{e}_{\alpha^{t}}$. In this case, $\operatorname{dim}(\mathbf{e}(\mathbf{c}))=\left(d_{1}, \ldots, d_{n}\right)$, where $\sum_{t=1}^{\nu} c_{t} \alpha^{t}=\sum_{i=1} d_{i} \alpha_{i}$, i.e. $\mathbf{c}$ is of i-homogeneity $\mathbf{d}$.

In particular, the classification of indecomposable modules is independent of the ground field.

Proof. Lus90a, sect. $4.12-4.15$ ]

Set $\left[\mathbf{V}, \mathbf{V}^{\prime}\right]=\operatorname{dim}_{F} \operatorname{Hom}_{\mathcal{Q}}\left(\mathbf{V}, \mathbf{V}^{\prime}\right)$ and $\left[\mathbf{V}, \mathbf{V}^{\prime}\right]^{1}=\operatorname{dim}_{F} \operatorname{Ext}_{\mathcal{Q}}^{1}\left(\mathbf{V}, \mathbf{V}^{\prime}\right)$. Note that $\operatorname{Hom}_{\mathcal{Q}}\left(\mathbf{V}, \mathbf{V}^{\prime}\right)$ is the $F$-vector space of morphisms $g: \mathbf{V} \rightarrow \mathbf{V}^{\prime}$ in $\operatorname{Mod}(\mathcal{Q})$ and $\operatorname{Ext}_{\mathcal{Q}}^{1}\left(\mathbf{V}, \mathbf{V}^{\prime}\right)$ is the $F$-vector space of extensions $0 \rightarrow \mathbf{V}^{\prime} \rightarrow \mathbf{E} \rightarrow \mathbf{V} \rightarrow 0$ in $\operatorname{Mod}(\mathcal{Q})$

For $\mathbf{d}=\left(d_{1}, \ldots, d_{n}\right) \in \mathbb{N}^{n}$, define

$$
E_{\mathbf{d}}=\bigoplus_{i \rightarrow j \in \mathcal{Q}^{1}} \operatorname{Hom}_{F}\left(F^{d_{i}}, F^{d_{j}}\right) \quad \text { and } \quad G_{\mathbf{d}}=\prod_{i=1}^{n} G L_{d_{i}}(F) .
$$

The group $G_{\mathbf{d}}$ acts on $E_{\mathbf{d}}$ by $(g \cdot f)_{i \rightarrow j}=\left(g_{j} f_{i j} g_{i}^{-1}\right)_{i \rightarrow j}$. An element of $E_{\mathbf{d}}$ can be seen as a module in $\operatorname{Mod}(\mathcal{Q})$ of dimension $\mathbf{d}$. Two elements of $E_{\mathbf{d}}$ define isomorphic modules if and only if they are in the same $G_{\mathbf{d}^{-}}$orbit. By theorem 2.3 there exists a bijection between the set of $\nu$-tuples $\mathbf{c}=\left(c_{1}, \ldots, c_{\nu}\right)$ of $\mathbf{i}$-homogeneity $\mathbf{d}$ and the 
set of $G_{\mathbf{d}^{-}}$-orbits in $E_{\mathbf{d}}$, where $\mathbf{c}=\left(c_{1}, \ldots, c_{\nu}\right)$ corresponds to the orbit $\mathcal{O}_{\mathbf{c}}$ whose elements are isomorphic to $\mathbf{e}(\mathbf{c})$.

There is a partial order on $\mathbb{N}^{\nu}$ given by $\mathbf{c}^{\prime} \preceq \mathbf{c}$ if $\mathbf{c}^{\prime}$ and $\mathbf{c}$ have the same $\mathbf{i}$ homogeneity and the orbit $\mathcal{O}_{\mathbf{c}^{\prime}}$ is contained in the Zariski closure $\overline{\mathcal{O}}_{\mathbf{c}}$ of $\mathcal{O}_{\mathbf{c}}$. This is the so-called degeneration ordering.

Let $\mathcal{S}$ be the set of non-split short exact sequences of modules of $\mathcal{Q}$ and let $O p$ be the subset of $\mathcal{S}$ consisting of all sequences for which the first and the last module are indecomposable. Hence if $\Upsilon \in O p$ then

$$
\Upsilon: 0 \rightarrow \mathbf{e}_{\alpha^{s}} \rightarrow \mathbf{V} \rightarrow \mathbf{e}_{\alpha^{t}} \rightarrow 0
$$

for some $s, t \in\{1, \ldots, \nu\}$ and some module $\mathbf{V}$. The elements of $O p$ are called elementary operations. For $\Upsilon: 0 \rightarrow \mathbf{e}_{\alpha^{s}} \rightarrow \mathbf{V} \rightarrow \mathbf{e}_{\alpha^{t}} \rightarrow 0 \in O p$ define $i n(\Upsilon)=s$ and $\operatorname{out}(\Upsilon)=t$ and denote by op ${ }^{\Upsilon}$ the vector $\left(o p_{1}^{\Upsilon}, \ldots, o p_{\nu}^{\Upsilon}\right) \in \mathbb{Z}^{\nu}$ given by

$$
o p_{r}^{\Upsilon}=\left\{\begin{array}{cl}
-1 & \text { if } r=s, t \\
1 & \text { if } \mathbf{e}_{\alpha^{r}} \text { is a direct summand of } \mathbf{V} \\
0 & \text { otherwise. }
\end{array}\right.
$$

For all $\mathbf{c} \in \mathbb{N}^{\nu}$ define $O p(\mathbf{c})=\left\{\Upsilon \in O p \mid \mathbf{c}+\mathbf{o p}^{\Upsilon} \in \mathbb{N}^{\nu}\right\}$. Thus an elementary operation $\Upsilon \in O p(\mathbf{c})$ allows us to go from one orbit $\mathcal{O}_{\mathbf{c}}$ to another orbit $\mathcal{O}_{\mathbf{c}+\mathbf{o p}^{\Upsilon}}$. As we will see in Theorem 2.4 below, elementary operations do not only preserve the $\mathbf{i}$-homogeneity but they are also compatible with the partial ordering $\preceq$.

The following theorem is shown in Bon95.

Theorem 2.4. Let $\mathbf{c}, \mathbf{c}^{\prime} \in \mathbb{N}^{\nu}$. Then the following four statements are equivalent:

(i) $\mathbf{c}^{\prime} \preceq \mathbf{c}$

(ii) There is a sequence of elementary operations $\Upsilon_{1}, \Upsilon_{2}, \ldots, \Upsilon_{k}$ such that $\Upsilon_{l} \in$ $O p\left(\mathbf{c}^{\prime}+\sum_{i=1}^{l-1} \mathbf{o p}^{\Upsilon_{i}}\right)$ and $\mathbf{c}^{\prime}+\sum_{i=1}^{k} \mathbf{o p}^{\Upsilon_{i}}=\mathbf{c}$

(iii) $\left[\mathbf{e}_{\alpha}, \mathbf{e}\left(\mathbf{c}^{\prime}\right)\right] \geq\left[\mathbf{e}_{\alpha}, \mathbf{e}(\mathbf{c})\right]$ for all indecomposable modules $\mathbf{e}_{\alpha}$.

(iv) $\left[\mathbf{e}\left(\mathbf{c}^{\prime}\right), \mathbf{e}_{\alpha}\right] \geq\left[\mathbf{e}(\mathbf{c}), \mathbf{e}_{\alpha}\right]$ for all indecomposable modules $\mathbf{e}_{\alpha}$.

2.4. Hall algebras. Fix a quiver $\mathcal{Q}$ and a $\nu$-tuple $\mathbf{i}$ adapted to $\mathcal{Q}$. Let $\mathcal{H}_{\mathcal{Q}}=\mathcal{H}$ be the twisted Hall algebra associated to the quiver $\mathcal{Q}$. $\mathcal{H}$ is the free $\mathbb{Q}(v)$-module with basis $\mathcal{B}$ the set of isomorphism classes of representations of the quiver $\mathcal{Q}$, with multiplication

$$
\left[\mathbf{e}\left(\mathbf{c}^{\prime}\right)\right] \cdot\left[\mathbf{e}\left(\mathbf{c}^{\prime \prime}\right)\right]=v^{<\mathbf{e}\left(\mathbf{c}^{\prime}\right), \mathbf{e}\left(\mathbf{c}^{\prime \prime}\right)>} \sum_{\mathbf{c} \in \mathbb{N}^{\nu}} F_{\mathbf{c}^{\prime}, \mathbf{c}^{\prime \prime}}^{\mathbf{c}}[\mathbf{e}(\mathbf{c})]
$$

where $<\mathbf{e}\left(\mathbf{c}^{\prime}\right), \mathbf{e}\left(\mathbf{c}^{\prime \prime}\right)>=\operatorname{dim}_{\mathbb{F}_{v^{2}}} \operatorname{Hom}\left(\mathbf{e}\left(\mathbf{c}^{\prime}\right), \mathbf{e}\left(\mathbf{c}^{\prime \prime}\right)\right)-\operatorname{dim}_{\mathbb{F}_{v^{2}}} \operatorname{Ext}\left(\mathbf{e}\left(\mathbf{c}^{\prime}\right), \mathbf{e}\left(\mathbf{c}^{\prime \prime}\right)\right)$ and $F_{\mathbf{c}^{\prime}, \mathbf{c}^{\prime \prime}}^{\mathbf{c}}$ is the number of submodules of $\mathbf{e}(\mathbf{c})$ that are isomorphic to $\mathbf{e}\left(\mathbf{c}^{\prime \prime}\right)$ and are such that the corresponding quotient module is isomorphic to $\mathbf{e}\left(\mathbf{c}^{\prime}\right)$, as representations over $\mathbb{F}_{v^{2}}$. This defines polynomials $F_{\mathbf{c}^{\prime}, \mathbf{c}^{\prime \prime}}^{\mathbf{c}}$ which are called Hall polynomials.

Theorem 2.5. There exists an isomorphism $\eta: \mathbf{U}^{+} \rightarrow \mathcal{H}$ of $\mathbb{Z}^{n}$-graded $\mathbb{Q}(v)$ algebras such that $\eta\left(E_{i}\right)=\left[\mathbf{e}_{\alpha_{i}}\right]$. It maps $E_{\mathbf{i}}^{\mathbf{c}}$ to $v^{[\mathbf{e}(\mathbf{c}), \mathbf{e}(\mathbf{c})]-\operatorname{Tr}\left(\operatorname{wt}\left(E_{\mathbf{i}}^{\mathbf{c}}\right)\right)}[\mathbf{e}(\mathbf{c})]$.

Proof. Rin93 Gre95.

We shall need the following corollary, see Cal :

Corollary 2.6. Up to a power of $v$, the $E_{\mathbf{i}}^{\mathbf{c}^{\prime}}$-coefficient of $\prod_{i=1}^{k} E_{\mathbf{i}}^{\mathbf{c}_{i}}$ is $F_{\mathbf{c}_{1}, \ldots, \mathbf{c}_{k}}^{\mathbf{c}^{\prime}}\left(v^{2}\right)$, where the polynomial $F_{\mathbf{c}_{1}, \ldots, \mathbf{c}_{k}}^{\mathbf{c}^{\prime}}(q)$ denotes the number of filtrations of $\mathbf{e}\left(\mathbf{c}^{\prime}\right)$ with successive quotients isomorphic to $\mathbf{e}\left(\mathbf{c}_{1}\right), \ldots, \mathbf{e}\left(\mathbf{c}_{k}\right)$ over $\mathbb{F}_{q}$. 
2.5. Coalgebra structure. Define an algebra structure on $\mathbf{U}^{+} \otimes_{\mathbb{Q}(v)} \mathbf{U}^{+}$by

$$
\left(x \otimes x^{\prime}\right) \cdot\left(y \otimes y^{\prime}\right)=v^{\left(\operatorname{wt}\left(x^{\prime}\right), \operatorname{wt}(y)\right)}(x y) \otimes\left(x^{\prime} y^{\prime}\right)
$$

on homogeneous elements. Let $\delta: \mathbf{U}^{+} \rightarrow \mathbf{U}^{+} \otimes_{\mathbb{Q}(v)} \mathbf{U}^{+}$be the $\mathbb{Q}(v)$-algebra map given by $\delta\left(E_{i}\right)=E_{i} \otimes 1+1 \otimes E_{i}$. Now, there exists a unique $\mathbb{Q}(v)$-bilinear form $\left(\right.$, ) on $\mathbf{U}^{+}$such that $\left(E_{i}, E_{j}\right)=\delta_{i, j}\left(1-v^{-2}\right)^{-1}$ and $\left(x, y y^{\prime}\right)=\left(\delta(x), y \otimes y^{\prime}\right)$, where $(,$,$) is extended to \mathbf{U}^{+} \otimes \mathbf{U}^{+}$by the rule $\left(x \otimes x^{\prime}, y \otimes y^{\prime}\right)=(x, y) .\left(x^{\prime}, y^{\prime}\right)$.

The Hall algebra approach of the quantum algebra $\mathbf{U}^{+}$gives a nice description of its coalgebra structure, Gre95. For every representation $\mathbf{e}(\mathbf{c})$ of the quiver $\mathcal{Q}$, let $a_{\mathbf{c}}\left(v^{2}\right)$ be the number of automorphisms of $\mathbf{e}(\mathbf{c})$ as a representation over $\mathbb{F}_{v^{2}}$. Recall Rin90, that

$$
\left.a_{\mathbf{c}}\left(v^{2}\right)=v^{2 \sum_{s<t} c_{s} c_{t}\left[\mathbf{e}_{\alpha^{s}}, \mathbf{e}_{\alpha^{t}}\right]} \prod_{s=1}^{\nu} \mid G L_{c_{s}}\left(\mathbb{F}_{v^{2}}\right)\right) \mid
$$

We have Rei99]:

$$
\left(E_{\mathbf{i}}^{\mathbf{c}}, E_{\mathbf{i}}^{\mathbf{c}^{\prime}}\right)=\delta_{\mathbf{c}, \mathbf{c}^{\prime}} v^{2[\mathbf{e}(\mathbf{c}), \mathbf{e}(\mathbf{c})]} a_{\mathbf{c}}\left(v^{2}\right)^{-1} .
$$

This proves that the basis $B_{\mathbf{i}}$ is orthogonal. We can define the dual basis for this pairing $B_{\mathbf{i}}^{*}=\left\{E_{\mathbf{i}}^{\mathbf{c} *}, \mathbf{c} \in \mathbb{N}^{\nu}\right\}$, with

$$
E_{\mathbf{i}}^{\mathbf{c} *}=v^{-2[\mathbf{e}(\mathbf{c}), \mathbf{e}(\mathbf{c})]} a_{\mathbf{c}}\left(v^{2}\right) E_{\mathbf{i}}^{\mathbf{c}} .
$$

This implies that the algebra $\mathbf{U}^{+}$is generated by $E_{\mathbf{i}}^{\mathbf{b}(s) *}, 1 \leq s \leq \nu$, with straightening relations :

$$
E_{\mathbf{i}}^{\mathbf{b}(t) *} E_{\mathbf{i}}^{\mathbf{b}(s) *}=\sum_{\mathbf{b}(t)+\mathbf{b}(s) \preceq \mathbf{c}} G_{\mathbf{b}(t), \mathbf{b}(s)}^{\mathbf{c}} E_{\mathbf{i}}^{\mathbf{c} *} .
$$

In order to understand those straightening relations, we have to define another polynomial. Let $0 \rightarrow \mathbf{e}\left(\mathbf{c}^{\prime}\right) \rightarrow \mathbf{e}(\mathbf{c}) \rightarrow \mathbf{e}\left(\mathbf{c}^{\prime \prime}\right) \rightarrow 0$ be a non-split exact sequence of representations of $\mathcal{Q}$. Then, the set of points $\mathcal{C}_{\mathbf{c}^{\prime \prime}, \mathbf{c}^{\prime}}^{\mathbf{c}}$ in the $\mathbb{F}_{v^{2}}$-space $\operatorname{Ext}^{1}\left(\mathbf{e}\left(\mathbf{c}^{\prime \prime}\right), \mathbf{e}\left(\mathbf{c}^{\prime}\right)\right)$ corresponding to this extension is a cone (minus the zero point). Set

$$
E_{\mathbf{c}^{\prime \prime}, \mathbf{c}^{\prime}}^{\mathbf{c}}\left(v^{2}\right):=\left|\mathcal{C}_{\mathbf{c}^{\prime \prime}, \mathbf{c}^{\prime}}^{\mathbf{c}}\right| \text {. }
$$

It is known that $E_{\mathbf{c}^{\prime \prime}, \mathbf{c}^{\prime}}^{\mathbf{c}}$ is a polynomial.

Proposition 2.7. Suppose $1 \leq s<t \leq \nu$. Then, for all $\mathbf{c}$ in $\mathbb{N}^{\nu}$ and up to a power of $v$, we have

$$
G_{\mathbf{b}(t), \mathbf{b}(s)}^{\mathbf{c}}= \begin{cases}1 & \text { if } \mathbf{c}=\mathbf{b}(s)+\mathbf{b}(t), \\ E_{\mathbf{b}(t), \mathbf{b}(s)}^{\mathbf{c}}\left(v^{2}\right) & \text { if } \mathbf{c}=\mathbf{b}(s)+\mathbf{b}(t)+\mathbf{o p}^{\Upsilon} \text { for some } \Upsilon \in O p, \\ 0 & \text { otherwise. }\end{cases}
$$

Proof. This is a direct consequence of (2.4) and the Riedtmann formula which states

$$
F_{\mathbf{b}(t), \mathbf{b}(s)}^{\mathbf{c}}\left(v^{2}\right) a_{\mathbf{b}(t)}\left(v^{2}\right) a_{\mathbf{b}(s)}\left(v^{2}\right) a_{\mathbf{c}}\left(v^{2}\right)^{-1}=E_{\mathbf{b}(t), \mathbf{b}(s)}^{\mathbf{c}}\left(v^{2}\right) .
$$

Remark 2.8. As the set $\mathcal{C}_{\mathbf{c}^{\prime \prime}, \mathbf{c}^{\prime}}^{\mathbf{c}} \cup\{0\}$ is a cone, we see that the polynomial $E_{\mathbf{c}^{\prime \prime}, \mathbf{c}^{\prime}}^{\mathbf{c}}\left(v^{2}\right)$ can be factorized by $v^{2}-1$. So, the proposition shows that the $\mathcal{A}$-space generated by the $E_{\mathbf{i}}^{\mathbf{c} *}$ is an algebra, and its specialization at $v=1$ is a (commutative) polynomial algebra. Indeed, it is known that this specialization is the algebra of regular functions on the maximal unipotent group. 
Now, let $\sigma$ be the $\mathbb{Q}$-antiautomorphism of $\mathbf{U}^{+}$such that $\sigma\left(E_{i}\right)=E_{i}, 1 \leq i \leq n$, $\sigma(v)=v^{-1}$.

Lemma 2.9. Let $x, y$ be homogeneous elements in $\mathbf{U}^{+}$, and let $t \in\{1, \ldots, \nu\}$. We have

(i) $\quad(\bar{x}, y)=(-v)^{\operatorname{Tr}(\operatorname{wt}(x))} v^{\frac{1}{2}(\operatorname{wt}(x), \operatorname{wt}(x))_{Q}} \overline{(x, \sigma(y))}$.

(ii) $\sigma\left(E_{\mathbf{i}}^{\mathbf{b}(t)}\right)=(-1)^{\operatorname{Tr}\left(\alpha^{t}\right)-1} E_{\mathbf{i}}^{\mathbf{b}(t)}$, up to a power of $v$.

Proof. (i) is a direct consequence of [Rei99 4.3] and (iii) is proved in Cal prop. 2.1].

2.6. Local intersection cohomology of orbit closures. In this subsection, let $\mathbb{F}$ be an algebraic closure of a finite field $\mathbb{F}_{q}$ with $q=p^{e}$ elements, where $p$ is a prime number, and let $\mathbf{d}=\left(d_{1}, \ldots, d_{n}\right) \in \mathbb{N}^{n}$. We will write the $\operatorname{dimension} \operatorname{dim}\left(\mathcal{O}_{\mathbf{c}}\right)$ of the orbit $\mathcal{O}_{\mathbf{c}}$ by $d(\mathbf{c})$.

The results of this subsection have been proved in [Lus90a chap. $9-10$ ].

Proposition 2.10. Let $\mathbf{c} \in \mathbb{N}^{\nu}$ be of $\mathbf{i}$-homogeneity $\mathbf{d}$. Then for each $\mathbf{c}^{\prime} \preceq \mathbf{c}$, there exists $\omega_{\mathbf{c}^{\prime}}^{\mathbf{c}} \in \mathbb{Z}\left[v, v^{-1}\right]$ such that

$$
\overline{E_{\mathbf{i}}^{\mathbf{c}}}=\sum_{\mathbf{c}^{\prime} \preceq \mathbf{c}} \omega_{\mathbf{c}^{\prime}}^{\mathbf{c}} E_{\mathbf{i}}^{\mathbf{c}^{\prime}} .
$$

Moreover $\omega_{\mathbf{c}}^{\mathbf{c}}=1$ and for all $\mathbf{c}^{\prime} \preceq \mathbf{c}, \Omega_{\mathbf{c}^{\prime}}^{\mathbf{c}} \stackrel{\text { def }}{=} v^{d(\mathbf{c})-d\left(\mathbf{c}^{\prime}\right)} \omega_{\mathbf{c}^{\prime}}^{\mathbf{c}}$ is an element of $\mathbb{Z}\left[v^{2}, v^{-2}\right]$.

Theorem 2.11. Let $\mathbf{c} \in \mathbb{N}^{\nu}$ be of $\mathbf{i}$-homogeneity $\mathbf{d}$ and let $\mathcal{E}^{\mathbf{c}} \in \mathbf{B}$ be the unique canonical basis element such that $\pi\left(\mathcal{E}^{\mathbf{c}}\right)=\pi\left(E_{\mathbf{i}}^{\mathbf{c}}\right)$. Then

(i) $\mathcal{E}^{\mathbf{c}}=\sum_{\mathbf{c}^{\prime}} \zeta_{\mathbf{c}^{\prime}}^{\mathbf{c}} E_{\mathbf{i}}^{\mathbf{c}^{\prime}}$, where $\mathbf{c}^{\prime}$ runs over the set of elements of $\mathbb{N}^{\nu}$ of $\mathbf{i}$ homogeneity $\mathbf{d}, \zeta_{\mathbf{c}}^{\mathbf{c}}=1$ and $\zeta_{\mathbf{c}^{\prime}}^{\mathbf{c}} \in v^{-1} \mathbb{Z}\left[v^{-1}\right]$ for $\mathbf{c}^{\prime} \neq \mathbf{c}$.

(ii) If $\mathbf{c}^{\prime} \npreceq \mathbf{c}$, then $\zeta_{\mathbf{c}^{\prime}}^{\mathbf{c}}=0$.

(iii) If $\overline{()}$ is the $\mathbb{Z}$-linear involution of $\mathbb{Z}\left[v, v^{-1}\right]$ sending $v$ to $v^{-1}$, then

$$
\zeta_{\mathbf{c}^{\prime}}^{\mathbf{c}}=\sum_{\substack{\mathbf{c}^{\prime \prime} \\ \mathbf{c}^{\prime} \preceq \mathbf{c}^{\prime \prime} \preceq \mathbf{c}}} w_{\mathbf{c}^{\prime}}^{\mathbf{c}^{\prime \prime}} \overline{\zeta_{\mathbf{c}^{\prime \prime}}^{\mathbf{c}}} .
$$

(iv) If $\mathbf{c}^{\prime} \preceq \mathbf{c}, f$ is a $\mathbb{F}_{q}$-rational point of the orbit $\mathcal{O}_{\mathbf{c}^{\prime}}$ in $E_{\mathbf{d}}$ and $\mathcal{H}_{f}^{a}$ is the stalk at $f$ of the a-th cohomology sheaf of the intersection cohomology complex of the Zariski closure $\overline{\mathcal{O}}_{\mathbf{c}}$ of $\mathcal{O}_{\mathbf{c}}$ with coefficients in $\overline{\mathbb{Q}_{\ell}}$ (extended by zero on the complement of that closure), where $\ell$ is a prime number $\neq p$, and with the $\mathbb{F}_{q}$-structure such that the Frobenius map acts as identity on the stalks of its 0-th cohomology sheaf at the rational points of the orbit $\mathcal{O}_{\mathbf{c}}$, then

$\mathcal{H}_{f}^{2 a+1}=0 \quad$ for all $a \quad$ and $\quad v^{d(\mathbf{c})-d\left(\mathbf{c}^{\prime}\right)} \zeta_{\mathbf{c}^{\prime}}^{\mathbf{c}}=\sum_{a} \operatorname{dim}\left(\mathcal{H}_{f}^{2 a}\right) v^{2 a}$.

In particular, $v^{d(\mathbf{c})-d\left(\mathbf{c}^{\prime}\right)} \zeta_{\mathbf{c}^{\prime}}^{\mathbf{c}}$ is a polynomial in $v^{2}$ with coefficients in $\mathbb{N}$.

Definition 2.12. We say that the orbit closure $\overline{\mathcal{O}}_{\mathbf{c}}$ is rationally smooth at $\mathcal{O}_{\mathbf{c}^{\prime}} \subset \overline{\mathcal{O}}_{\mathbf{c}}$ if for all $\mathbf{c}^{\prime \prime}$ such that $\mathbf{c}^{\prime} \preceq \mathbf{c}^{\prime \prime} \preceq \mathbf{c}$ we have $\sum_{a} \operatorname{dim}\left(\mathcal{H}_{f}^{2 a}\right) v^{2 a}=1$ for a $\mathbb{F}_{q}$-rational point $f \in \mathcal{O}_{\mathbf{c}^{\prime \prime}}$, i.e. if $\zeta_{\mathbf{c}^{\prime \prime}}^{\mathbf{c}}=v^{d\left(\mathbf{c}^{\prime \prime}\right)-d(\mathbf{c})}$ for all $\mathbf{c}^{\prime \prime}$ such that $\mathbf{c}^{\prime} \preceq \mathbf{c}^{\prime \prime} \preceq \mathbf{c}$.

The orbit closure $\overline{\mathcal{O}}_{\mathbf{c}}$ is rationally smooth if it is rationally smooth at each $\mathcal{O}_{\mathbf{c}^{\prime}} \subset \overline{\mathcal{O}}_{\mathbf{c}}$, i.e. if $\zeta_{\mathbf{c}^{\prime}}^{\mathbf{c}}=v^{d\left(\mathbf{c}^{\prime}\right)-d(\mathbf{c})}$ for all $\mathbf{c}^{\prime} \preceq \mathbf{c}$. 
Remark 2.13. By a result of $[\mathrm{BM}], \overline{\mathcal{O}}_{\mathbf{c}}$ is rationally smooth iff for each point $x \in \overline{\mathcal{O}}_{\mathbf{c}}$ we have

$$
H^{i}\left(\overline{\mathcal{O}}_{\mathbf{c}}, \overline{\mathcal{O}}_{\mathbf{c}} \backslash\{x\} ; \mathbb{Q}\right)= \begin{cases}\mathbb{Q} & \text { if } i=2 \operatorname{dim} \overline{\mathcal{O}}_{\mathbf{c}} \\ 0 & \text { otherwise }\end{cases}
$$

where $H^{i}$ denotes ordinary cohomology.

\section{Algebraic Approach}

This section gives a generalization of results in [BS]. We first prove some formulas on the coefficients $\Omega_{\mathbf{c}^{\prime}}^{\mathbf{c}}$.

3.1. A formula for $\Omega_{\mathbf{c}^{\prime}}^{\mathbf{c}}$. We fix a quiver $\mathcal{Q}$ and a $\nu$-tuple $\mathbf{i}$ adapted to $\mathcal{Q}$ as in the previous section. For all $\mathbf{c}$ in $\mathbb{N}^{\nu}$, set $\mathbf{c}^{t}:=c_{t} \mathbf{b}(t), 1 \leq t \leq \nu$. An explicit formula for $\Omega_{\mathbf{c}^{\prime}}^{\mathbf{c}}$ can be calculated :

Proposition 3.1. For every $\mathbf{c}, \mathbf{c}^{\prime}$ in $\mathbb{N}^{\nu}$, we have :

$$
\Omega_{\mathbf{c}^{\prime}}^{\mathbf{c}}=F_{\mathbf{c}^{\prime}, \ldots, \mathbf{c}^{\prime 1}}^{\mathbf{c}}\left(v^{-2}\right)\left(\prod_{t=1}^{\nu} a_{\mathbf{c}^{\prime t}}\left(v^{-2}\right)\right) a_{\mathbf{c}}\left(v^{-2}\right)^{-1}
$$

up to a power of $v$.

Proof. In the following proof, equalities are up to a power of $v$. We first write $\Omega_{\mathbf{c}^{\prime}}^{\mathbf{c}}=$ $\left(\overline{E_{\mathbf{i}}^{\mathbf{c}}}, E_{\mathbf{i}}^{\mathbf{c}^{\prime} *}\right)$. Hence, by Lemma 2.9 (i), we have $\overline{\Omega_{\mathbf{c}^{\prime}}^{\mathbf{c}}}=(-1)^{\operatorname{Tr}\left(\operatorname{wt}\left(E_{\mathbf{i}}^{\mathbf{c}^{\prime}}\right)\right)}\left(E_{\mathbf{i}}^{\mathbf{c}}, \sigma\left(E_{\mathbf{i}}^{\mathbf{c}^{\prime} *}\right)\right)$. So, by formula (2.3), it remains to find the coefficient of $E_{\mathbf{i}}^{\mathbf{c}}$ in the decomposition of $\sigma\left(E_{\mathbf{i}}^{\mathbf{c}^{\prime} *}\right)$ in the basis $B_{\mathbf{i}}$.

It is easily seen that $E_{\mathbf{i}}^{\mathbf{c}^{\prime} *}=\prod_{t=1}^{\nu} E_{\mathbf{i}}^{c_{t}^{\prime} \mathbf{b}(t) *}$, up to a power of $v$. Then, using equation (2.4), Lemma 2.9 (ii) and the fact that $\overline{a_{c_{t} \mathbf{b}(t)}\left(v^{2}\right)} a_{c_{t} \mathbf{b}(t)}\left(v^{2}\right)^{-1}=(-1)^{c_{t}}$ up to a power of $v$, we get

$$
\overline{\Omega_{\mathbf{c}^{\prime}}^{\mathbf{c}}}=\left(E_{\mathbf{i}}^{\mathbf{c}}, \prod_{t=\nu}^{1} E_{\mathbf{i}}^{c_{t}^{\prime} \mathbf{b}(t) *}\right) \text { up to a power of } v .
$$

Finally, using equation 2.4 Corollary 2.6 (i) and equation 2.3 we obtain :

$$
\overline{\Omega_{\mathbf{c}^{\prime}}^{\mathbf{c}}}=F_{\mathbf{c}^{\prime}, \ldots, \mathbf{c}^{\prime 1}}^{\mathbf{c}}\left(v^{2}\right)\left(\prod_{t=1}^{\nu} a_{\mathbf{c}^{\prime t}}\left(v^{2}\right)\right) a_{\mathbf{c}}\left(v^{2}\right)^{-1}
$$

and the proposition follows.

There is a nice particular case of this formula when $\mathbf{e}\left(\mathbf{c}^{\prime}\right)$ is the sum of two indecomposable modules.

Corollary 3.2. Suppose $\mathbf{c}^{\prime}=\mathbf{b}(s)+\mathbf{b}(t), s<t$. Then, up to a power of $v$,

$$
\Omega_{\mathbf{c}^{\prime}}^{\mathbf{c}}=E_{\mathbf{b}(t), \mathbf{b}(s)}^{\mathbf{c}}\left(v^{-2}\right) .
$$

Proof. This is a direct application of the Riedtmann formula, Rie94. 
3.2. Derivative of $\Omega_{\mathbf{c}^{\prime}}^{\mathbf{c}}$ at $v=1$. Set

$$
e_{\mathbf{c}^{\prime \prime}, \mathbf{c}^{\prime}}^{\mathbf{c}}\left(v^{2}\right):=\frac{E_{\mathbf{c}^{\prime \prime}, \mathbf{c}^{\prime}}^{\mathbf{c}}\left(v^{2}\right)}{v^{2}-1}
$$

which is a polynomial. Let $O p r$ be the set of regular elementary operations defined by

$$
O p r=\left\{\Upsilon: 0 \rightarrow \mathbf{e}_{\alpha^{s}} \rightarrow \mathbf{e}(\mathbf{c}) \rightarrow \mathbf{e}_{\alpha^{t}} \rightarrow 0, e_{\mathbf{b}(t), \mathbf{b}(s)}^{\mathbf{c}}(1) \neq 0\right\} .
$$

With the notation above, we set $e^{\Upsilon}:=e_{\mathbf{b}(t), \mathbf{b}(s)}^{\mathbf{c}}(1)$.

By $\mathbb{N o ̈ r}$, we know that all elementary operations are regular in case A and D. This is not true in case E, see remark below. For $\mathbf{c}$ in $\mathbb{N}^{\nu}$, define $\operatorname{Opr}(\mathbf{c}):=$ Opr $\cap O p(\mathbf{c})$. We can now generalize [BS, Theorem 5.4].

Theorem 3.3. Let $\mathbf{c}, \mathbf{c}^{\prime}$ in $\mathbb{N}^{\nu}$ with same $\mathbf{i}$-homogeneity, then

(i) $\Omega_{\mathbf{c}}^{\mathbf{c}}=1$ and $\Omega_{\mathbf{c}^{\prime}}^{\mathbf{c}}(1)=0$ if $\mathbf{c}^{\prime} \prec \mathbf{c}$.

(ii) $\left.\frac{\mathrm{d}}{\mathrm{dv}} \Omega_{\mathbf{c}^{\prime}}^{\mathbf{c}}\right|_{v=1} \neq 0$ if and only if there exists $\Upsilon$ in $\operatorname{Opr}(\mathbf{c})$ such that $\mathbf{c}=\mathbf{c}^{\prime}+\mathbf{o p}^{\Upsilon}$.

(iii) If the condition in (ii) is verified, then $\left.\frac{\mathrm{d}}{\mathrm{dv}} \Omega_{\mathbf{c}^{\prime}}^{\mathbf{c}}\right|_{v=1}=-2 c_{s}^{\prime} c_{t}^{\prime} e^{\Upsilon}$, where $s=\operatorname{in}(\Upsilon)$ and $t=\operatorname{out}(\Upsilon)$.

Proof. The first formula of (i) is given in Proposition 2.10 For the second formula, just remark that the map $\overline{()}$ specializes on identity at $v=1$.

Let's prove (ii) and (iii). By equation (3.2) $\overline{\Omega_{\mathbf{c}^{\prime}}^{\mathbf{c}}}=\left(E_{\mathbf{i}}^{\mathbf{c}}, \prod_{t=\nu}^{1} E_{\mathbf{i}}^{c_{t}^{\prime} \mathbf{b}(t) *}\right)$, up to a power of $v$. Say $\sum_{\mathbf{c}^{\prime \prime}} h_{\mathbf{c}^{\prime}}^{\mathbf{c}^{\prime \prime}} E_{\mathbf{i}}^{\mathbf{c}^{\prime \prime} *}=\prod_{t=\nu}^{1} E_{\mathbf{i}}^{c_{t}^{\prime} \mathbf{b}(t) *}$ is the expansion of $\prod_{t=\nu}^{1} E_{\mathbf{i}}^{c_{t}^{\prime} \mathbf{b}(t) *}$ in the dual PBW-basis, thus $\overline{\Omega_{\mathbf{c}^{\prime}}^{\mathbf{c}}}=h_{\mathbf{c}^{\prime}}^{\mathbf{c}}$ up to a power of $v$. Then, since $\Omega_{\mathbf{c}^{\prime}}^{\mathbf{c}}(1)=0$, we have $\left.\frac{\mathrm{d}}{\mathrm{dv}} \overline{\Omega_{\mathbf{c}^{\prime}}}\right|_{v=1}=\left.\frac{\mathrm{d}}{\mathrm{dv}} h_{\mathbf{c}^{\prime}}^{\mathbf{c}}\right|_{v=1}$, which is non-zero iff the multiplicity of $(v-1)$ in $h_{\mathbf{c}^{\prime}}^{\mathbf{c}}$ is one. The above expansion is obtained by performing a certain number of straightening relations (2.5). By proposition 2.7 we have

(1) $h_{\mathbf{c}^{\prime}}^{\mathbf{c}^{\prime}}=1$, up to a power of $v$.

(2) If $\mathbf{c}=\mathbf{c}^{\prime}+\mathbf{o p}^{\Upsilon}$ for some $\Upsilon \in O p$ with $s=\operatorname{in}(\Upsilon), t=\operatorname{out}(\Upsilon)$, then

$$
h_{\mathbf{c}^{\prime}}^{\mathbf{c}}=E_{\mathbf{b}(t), \mathbf{b}(s)}^{\mathbf{c}}\left(v^{2}\right)=\left(v^{2}-1\right) e_{\mathbf{b}(t), \mathbf{b}(s)}^{\mathbf{c}}\left(v^{2}\right)
$$

up to a power of $v$. Then the multiplicity of $(v-1)$ in $h_{\mathbf{c}^{\prime}}^{\mathbf{c}}$ is at least one and is equal to one iff $\Upsilon$ is regular.

(3) All other $h_{\mathbf{c}^{\prime}}^{\mathbf{c}^{\prime \prime}}$ have $(v-1)$ multiplicity at least 2 .

This proves (ii). Now let $\mathbf{c}=\mathbf{c}^{\prime}+\mathbf{o p}^{\Upsilon}$ with $\Upsilon \in O p r$ and $s=i n(\Upsilon), t=\operatorname{out}(\Upsilon)$. Then the only way $E_{\mathbf{i}}^{\mathbf{c} *}$ terms appear in our expansion is in the straightening relations involving both $E_{\mathbf{i}}^{\mathbf{b}(t) *}$ and $E_{\mathbf{i}}^{\mathbf{b}(s) *}$. The number of these straightening relations is $c_{s}^{\prime} c_{t}^{\prime}$. Thus, by Proposition $2.7 h_{\mathbf{c}^{\prime}}^{\mathbf{c}}$ is a sum of $c_{s}^{\prime} c_{t}^{\prime}$ terms equal to $\left(v^{2}-1\right) e_{\mathbf{b}(t), \mathbf{b}(s)}^{\mathbf{c}}\left(v^{2}\right)$ up to a power of $v$. Hence, $\left.\frac{\mathrm{d}}{\mathrm{dv}} h_{\mathbf{c}^{\prime}}^{\mathbf{c}}\right|_{v=1}=2 c_{s}^{\prime} c_{t}^{\prime} e^{\Upsilon}$. The theorem follows since $\left.\frac{\mathrm{d}}{\mathrm{dv}} \Omega_{\mathbf{c}^{\prime}}^{\mathbf{c}}\right|_{v=1}=-\left.\frac{\mathrm{d}}{\mathrm{dv}} \overline{\Omega_{\mathbf{c}^{\prime}}}\right|_{v=1}$.

Remark 3.4. The formula in (iii) slightly differs from the one in BS because of the factor $e^{\Upsilon}$. This factor can be obtained from the polynomials calculated in №̈r. In type $\mathrm{A}$, it can only be 1 , so this agrees with the formula in $\mathrm{BS}$. In type $\mathrm{D}$ this constant can be equal to 1 or -1 . In type $\mathrm{E}$, it can be equal to $1,-1$ and 0 . 
3.3. Rational Smoothness. In this section, we will characterize which orbit closures $\overline{\mathcal{O}_{\mathbf{c}}}$ are rationally smooth. As a consequence, we will show that if $\overline{\mathcal{O}_{\mathbf{c}}}$ is rationally smooth, then $\overline{\mathcal{O}_{c}}$ is smooth.

Let $u=v^{2}$. The next proposition has been shown in BS, Cor 6.4]. We sketch a proof of it for completeness.

Suppose that $\overline{\mathcal{O}_{\mathbf{c}}}$ is rationally smooth, then for all $\mathbf{c}^{\prime} \preceq \mathbf{c}$ we have $\zeta_{\mathbf{c}^{\prime}}^{\mathbf{c}}=v^{d\left(\mathbf{c}^{\prime}\right)-d(\mathbf{c})}$. Fix now $\mathbf{c}^{\prime} \preceq \mathbf{c}$. We obtain by Theorem 2.11 (iii) that

$$
\sum_{\mathbf{c}^{\prime} \preceq \mathbf{c}^{\prime \prime} \preceq \mathbf{c}} \Omega_{\mathbf{c}^{\prime}}^{\mathbf{c}^{\prime \prime}} u^{d(\mathbf{c})-d\left(\mathbf{c}^{\prime \prime}\right)}=1 .
$$

By taking the derivative to $u$ evaluated at $u=1$, we get by Theorem 3.3 (i) :

Proposition 3.5. $\overline{\mathcal{O}_{\mathbf{c}}}$ is rationally smooth then

$$
-\left.\sum_{\substack{\mathbf{c}^{\prime \prime} \\ \mathbf{c}^{\prime} \prec \mathbf{c}^{\prime \prime} \preceq \mathbf{c}}} \frac{\mathrm{d} \Omega_{\mathbf{c}^{\prime}}^{\mathbf{c}^{\prime \prime}}}{\mathrm{du}}\right|_{u=1}=d(\mathbf{c})-d\left(\mathbf{c}^{\prime}\right)
$$

for all $\mathbf{c}^{\prime} \preceq \mathbf{c}$.

For a subset $J$ of the set $\mathcal{Q}^{1}$ of arrows of the quiver $\mathcal{Q}$, we define

$$
E_{\mathbf{d}}(J)=\left\{\left(f_{i j}\right)_{i \rightarrow j} \in E_{\mathbf{d}} \mid f_{i j}=0 \quad \text { if }\{i, j\} \in \mathcal{Q}^{1} \backslash J\right\} .
$$

The following properties are easily proved and left to the reader. Let $J, J^{\prime}$ be two subsets of the set $\mathcal{Q}^{1}$ of edges of $\mathcal{Q}$.

(1) $E_{\mathbf{d}}(J)$ is a linear subspace of $E_{\mathbf{d}}$ of dimension $\operatorname{dim}\left(E_{\mathbf{d}}(J)\right)=\sum_{\{i, j\} \in J} d_{i} d_{j}$. In particular, $E_{\mathbf{d}}(J)$ is a smooth variety.

(2) $E_{\mathbf{d}}(J)$ is a $G_{\mathbf{d}^{-}}$-stable closed subset of $E_{\mathbf{d}}$ and it is a finite union of $G_{\mathbf{d}^{-}}$ orbits. As a consequence and because the field $\mathbb{F}$ is algebraically closed, we get that there is a unique open dense $G_{\mathbf{d}^{-}}$-orbit in $E_{\mathbf{d}}(J)$. We will denote this orbit by $\mathcal{O}(J)$.

(3) $E_{\mathbf{d}}(J) \cap E_{\mathbf{d}}\left(J^{\prime}\right)=E_{\mathbf{d}}\left(J \cap J^{\prime}\right)$ and $E_{\mathbf{d}}(J)+E_{\mathbf{d}}\left(J^{\prime}\right)=E_{\mathbf{d}}\left(J \cup J^{\prime}\right)$.

(4) $E_{\mathbf{d}}(J) \subseteq E_{\mathbf{d}}\left(J^{\prime}\right)$ if $J \subseteq J^{\prime}$.

(5) $E_{\mathbf{d}}(\emptyset)=\{0\}$ and $E_{\mathbf{d}}\left(\mathcal{Q}^{1}\right)=E_{\mathbf{d}}$.

Because of (3) and (5) we see that for each $G_{\mathbf{d}}$-orbit $\mathcal{O}_{\mathbf{c}}$ in $E_{\mathbf{d}}$, there is a unique smallest subset $J(\mathbf{c})$ of $\mathcal{Q}^{1}$ for which $\mathcal{O}_{\mathbf{c}} \subseteq E_{\mathbf{d}}(J(\mathbf{c}))$. In fact

$$
J(\mathbf{c})=\bigcap_{\mathcal{O}_{\mathbf{c}} \subseteq E_{\mathbf{d}}(J)} J .
$$

Because $E_{\mathbf{d}}(J)$ is closed, then $\mathcal{O}_{\mathbf{c}} \subseteq E_{\mathbf{d}}(J)$ if and only if $\overline{\mathcal{O}_{\mathbf{c}}} \subseteq E_{\mathbf{d}}(J)$. As a consequence, if $\mathbf{c}^{\prime \prime} \preceq \mathbf{c}$, then we get easily that $J\left(\mathbf{c}^{\prime \prime}\right) \subseteq J(\mathbf{c})$. Note also that we don't necessarily have $\mathcal{O}_{\mathbf{c}}=\mathcal{O}(J(\mathbf{c}))$. In fact, we will prove in theorem 3.6 that we get this equality precisely when $\overline{\mathcal{O}_{\mathbf{c}}}$ is rationally smooth.

Let $\mathbf{c}^{\mathrm{min}}=\left(c_{1}^{\min }, c_{2}^{\min }, \ldots, c_{\nu}^{\min }\right) \in \mathbb{N}^{\nu}$ be defined by

$$
c_{k}^{\min }= \begin{cases}d_{i} & \text { if } \alpha^{k}=\alpha_{i} \text { for some } i, 1 \leq i \leq n \\ 0 & \text { otherwise. }\end{cases}
$$

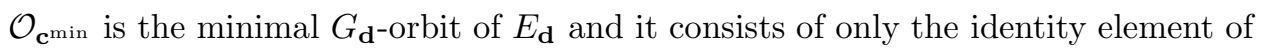
$E_{\mathbf{d}}$. 
Theorem 3.6. $\quad$ (1) We have the equality

$$
-\left.\sum_{\substack{\mathbf{c}^{\prime \prime} \\ \mathbf{c}^{\min } \prec \mathbf{c}^{\prime \prime} \preceq \mathbf{c}}} \frac{\mathrm{d} \Omega_{\mathbf{c}^{\min }}^{\mathbf{c}^{\prime \prime}}}{\mathrm{du}}\right|_{u=1}=\operatorname{dim}\left(E_{\mathbf{d}}(J(c))\right) .
$$

(2) $\overline{\mathcal{O}_{\mathbf{c}}}$ is rationally smooth if and only if $\overline{\mathcal{O}_{\mathbf{c}}}=E_{\mathbf{d}}(J(\mathbf{c}))$.

Proof. By theorem 3.3

$$
-\left.\sum_{\substack{\mathbf{c}^{\prime \prime} \\ \mathbf{c}^{\min } \prec \mathbf{c}^{\prime \prime} \preceq \mathbf{c}}} \frac{\mathrm{d} \Omega_{\mathbf{c}^{\min }}^{\mathbf{c}^{\prime \prime}}}{\mathrm{du}}\right|_{u=1}=\sum_{\substack{\Upsilon \in O p r\left(\mathbf{c}^{\min }\right) \\ \mathbf{c}^{\min }+\mathbf{p o p}^{\Upsilon} \preceq \mathbf{c}}} c_{i n(\Upsilon)}^{\min } c_{\text {out }(\Upsilon)}^{\min } e^{\Upsilon} .
$$

Now $\Upsilon \in O p r\left(\mathbf{c}^{\min }\right)$ iff $\Upsilon \in O p r$ and $c_{i n(\Upsilon)}^{\min } \neq 0 \neq c_{\text {out }(\Upsilon)}^{\min }$, i.e. $\quad \alpha^{i n(\Upsilon)}=\alpha_{j}$ and $\alpha^{\text {out }(\Upsilon)}=\alpha_{i}$ are simple roots, $c_{i n(\Upsilon)}^{\min }=d_{j}, c_{\text {out }(\Upsilon)}^{\min }=d_{i}$ and there is an arrow $i \rightarrow j \in \mathcal{Q}^{1}$. Moreover $\Upsilon: 0 \rightarrow \mathbf{e}_{\alpha_{j}} \rightarrow \mathbf{e}_{\alpha_{i}+\alpha_{j}} \rightarrow \mathbf{e}_{\alpha_{i}} \rightarrow 0$ and $e^{\Upsilon}=1$. Note also that in this situation $\mathbf{c}^{\text {min }}+\mathbf{o p}^{\Upsilon} \preceq \mathbf{c}$ iff $i \rightarrow j \in J(\mathbf{c})$. Thus

$$
-\left.\sum_{\substack{\mathbf{c}^{\prime \prime} \\ \mathbf{c}^{\min } \prec \mathbf{c}^{\prime \prime} \preceq \mathbf{c}}} \frac{\mathrm{d} \Omega_{\mathbf{c}^{\min }}^{\mathbf{c}^{\prime \prime}}}{\mathrm{du}}\right|_{u=1}=\sum_{i \rightarrow j \in J(\mathbf{c})} d_{i} d_{j}=\operatorname{dim}\left(E_{\mathbf{d}}(J(\mathbf{c}))\right),
$$

this proves (1).

(2) $E_{\mathbf{d}}(J(\mathbf{c}))$ is smooth, hence rationally smooth.

Conversely if $\overline{\mathcal{O}_{\mathbf{c}}}$ is rationally smooth, then applying proposition 3.5 for $\mathbf{c}^{\prime}=\mathbf{c}^{\text {min }}$ and using part (1), we get $\operatorname{dim}\left(E_{\mathbf{d}}(J(\mathbf{c}))\right)=d(\mathbf{c})$. Hence $\mathcal{O}_{\mathbf{c}}$ is the unique dense orbit in $E_{\mathbf{d}}(J(\mathbf{c}))$, thus $\overline{\mathcal{O}_{\mathbf{c}}}=E_{\mathbf{d}}(J(\mathbf{c}))$.

Corollary 3.7. An orbit closure of type $A, D, E$ is smooth, if and only if it is rationally smooth.

\section{Geometric approach.}

We present in this section an independent proof for Theorem 3.6 The interest of this proof is that we give an homological realization of the constants calculated in the previous section. To be more precise, the derivative of a coefficient $\Omega$ at $u=1$ can be seen as an Euler-Poincaré characteristic of a complex variety. The principle on which the alternative proof works is that if a cone $X$ in $\mathbb{C}^{n}$ is rationally smooth, then, the rational cohomology of the projectivization $\mathbb{P}(X)$ in $\mathbb{P}^{n-1}$ is the same as the rational cohomology of $\mathbb{P}^{d}$ where $d=\operatorname{dim} \mathbb{P}(X)$.

4.1. Euler-Poincaré characteristic. In the following section, the varieties $\overline{\mathcal{O}}$ are considered on the field $\mathbb{C}$. We fix $\mathbf{c} \in \mathbb{N}^{\nu}$ of $\mathbf{i}$-homogeneity $\mathbf{d}$. Set $X_{\mathbf{c}}:=\mathbb{P}\left(\overline{\mathcal{O}_{\mathbf{c}}} \backslash\{0\}\right)$, as a complex variety and let $X_{\mathbf{c}}\left(\mathbb{F}_{q}\right)$, resp. $X_{\mathbf{c}}\left(\overline{\mathbb{F}_{q}}\right)$, be the corresponding variety on the finite field $\mathbb{F}_{q}$, resp $\overline{\mathbb{F}_{q}}$. We know that there exists a discrete valuation ring $R \subset \mathbb{C}$ with residue field of characteristic $p$ and a variety $\hat{X}_{\mathbf{c}}$ defined on $R$ such that we get the variety $X_{\mathbf{c}}\left(\overline{\mathbb{F}_{q}}\right)$ over $\overline{\mathbb{F}_{q}}$ and the variety $X_{\mathbf{c}}$ over $\mathbb{C}$ by the base change to Spec $\overline{\mathbb{F}_{q}}$ and Spec $\mathbb{C}$ respectively.

Let $\chi\left(X_{\mathbf{c}}\right)$ be the Euler-Poincaré characteristic of the variety $X_{\mathbf{c}}$. Then

Theorem 4.1. For every $\mathbf{c} \in \mathbb{N}^{\nu}$ of $\mathbf{i}$-homogeneity $\mathbf{d}$ we have

$$
\chi\left(X_{\mathbf{c}}\right)=\operatorname{dim} E_{\mathbf{d}}(J(\mathbf{c})) .
$$


Proof. We first transpose the calculation of $\chi\left(X_{\mathbf{c}}\right)$ in the context of $l$-adic cohomology with compact support. We have

$$
\operatorname{dim}_{\mathbb{Q}} H^{i}\left(X_{\mathbf{c}}, \mathbb{Q}\right)=\operatorname{dim}_{\mathbb{C}} H^{i}\left(X_{\mathbf{c}}, \mathbb{C}\right)=\operatorname{dim}_{\mathbb{C}} H_{c}^{i}\left(X_{\mathbf{c}}, \mathbb{C}\right)=\operatorname{dim}_{\overline{\mathbb{Q}}_{\ell}} H_{c}^{i}\left(X_{\mathbf{c}}\left(\overline{\mathbb{F}_{q}}\right), \overline{\mathbb{Q}}_{\ell}\right),
$$

where $l$ is prime to $p$. Hence,

$$
\chi\left(X_{\mathbf{c}}\right)=\sum_{i}(-1)^{i} \operatorname{dim} H_{c}^{i}\left(X_{\mathbf{c}}\left(\overline{\mathbb{F}_{q}}\right), \overline{\mathbb{Q}}_{\ell}\right) .
$$

Fix a prime number $p$ such that $l$ is prime to $p$. We know that there is an action of the Frobenius Fr on the $l$-adic cohomology. By the Grothendieck trace formula, Dan96. 7.10], we know that

$$
\left|X_{\mathbf{c}}\left(\mathbb{F}_{p^{e}}\right)\right|=\sum_{i=0}^{2 \operatorname{dim} X_{\mathbf{c}}}(-1)^{i} \operatorname{tr}\left(\operatorname{Fr}^{e}, H_{c}^{i}\left(X_{\mathbf{c}}\left(\overline{\mathbb{F}_{q}}\right), \overline{\mathbb{Q}}_{\ell}\right)\right) .
$$

Now, the theorem of Deligne, Dan96, 8.21], asserts that $H_{c}^{i}\left(X_{\mathbf{c}}\left(\overline{\mathbb{F}_{q}}\right), \overline{\mathbb{Q}}_{\ell}\right)$ is filtered by Fr-stable subspace $W_{i, j}$ such that the eigenvalues of the Frobenius Fr on the successive quotients are powers of $p$ up to a root of one. So, there exists an $N$ such that if $e$ is a multiple of $N$, the eigenvalues of $\mathrm{Fr}^{e}$ are powers of $p^{e}$. For these $e$, we have

$$
\left|X_{\mathbf{c}}\left(\mathbb{F}_{p^{e}}\right)\right|=\sum_{i, j}(-1)^{i} p^{e k_{i, j}} \operatorname{dim}\left(W_{i, j} / W_{i, j-1}\right) .
$$

Now, we know that $\left|X_{\mathbf{c}}\left(\mathbb{F}_{q}\right)\right|$ is a polynomial $P_{\mathbf{c}}(q)$. So, the previous equation provides a polynomial equality which is true for an infinite number of $p^{e}$.

Hence, $\left|X_{\mathbf{c}}\left(\mathbb{F}_{q}\right)\right|=\sum_{i, j}(-1)^{i} q^{k_{i, j}} \operatorname{dim}\left(W_{i, j} / W_{i, j-1}\right)$, which implies by (4.1) that $\chi\left(X_{\mathbf{c}}\right)=P_{\mathbf{c}}(1)$. The proof of the theorem relies now on the following lemma.

Lemma 4.2. For every $\mathbf{c} \in \mathbb{N}^{\nu}$ of $\mathbf{i}$-homogeneity $\mathbf{d}$ we have

$$
P_{\mathbf{c}}(1)=\operatorname{dim}\left(E_{\mathbf{d}}(J(\mathbf{c}))\right) \text {. }
$$

Proof. First of all, let $\mathbf{S}_{\mathbf{c}}$ be the set of elements $\mathbf{c}^{\prime}$ of $\mathbf{i}$-homogeneity $\mathbf{d}$ such that there exists $\Upsilon \in O p$ such that $\mathbf{c}^{\text {min }}+\mathbf{o p}^{\Upsilon}=\mathbf{c}^{\prime} \preceq \mathbf{c}$. We have :

$$
\mathbf{S}_{\mathbf{c}}=\left\{\mathbf{c}^{i j},(i, j) \in J(\mathbf{c})\right\}
$$

with

$$
c_{s}^{i j}= \begin{cases}1 & \text { if } \alpha^{s}=\alpha_{i}+\alpha_{j} \\ d_{i}-1 & \text { if } \alpha^{s}=\alpha_{i} \\ d_{j}-1 & \text { if } \alpha^{s}=\alpha_{j} \\ 0 & \text { otherwise }\end{cases}
$$

We want to calculate $P_{\mathbf{c}}(q)$. As $\overline{\mathcal{O}_{\mathbf{c}}}$ is a disjoint union of orbits $\mathcal{O}_{\mathbf{c}^{\prime}}$ with $\mathbf{c}^{\prime} \preceq \mathbf{c}$, we have to count the cardinality of the set of $\mathbb{F}_{q}$-rational points $\mathcal{O}_{\mathbf{c}^{\prime}}\left(\mathbb{F}_{q}\right)$. This will be denoted by $Q_{\mathbf{c}^{\prime}}(q)$, thus $P_{\mathbf{c}}(q)=\frac{1}{q-1} \sum_{\mathbf{c}^{\min } \prec \mathbf{c}^{\prime} \prec \mathbf{c}} Q_{\mathbf{c}^{\prime}}(q)$. Let $x^{\prime}$ be a point of $\mathcal{O}_{\mathbf{c}^{\prime}}\left(\mathbb{F}_{q}\right)$ and let $G_{\mathbf{d}, x^{\prime}}$ be the isotropy group of $x^{\prime}$ in $G_{\mathbf{d}}$. Then, $Q_{\mathbf{c}^{\prime}}(q)=\frac{\left|G_{\mathbf{d}}\right|}{\left|G_{\mathbf{d}, x^{\prime}}\right|}$ and it is known that $G_{\mathbf{d}, x^{\prime}}$ is the group of automorphism of the module $\mathbf{e}\left(\mathbf{c}^{\prime}\right)$. Hence, $\left|G_{\mathbf{d}, x^{\prime}}\right|=a_{\mathbf{c}^{\prime}}(q)$ is given by (2.2). In particular, the multiplicity of $(q-1)$ in $Q_{\mathbf{c}^{\prime}}(q)$ is $\sum_{i=1}^{n} d_{i}-\sum_{s=1}^{\nu} c_{s}^{\prime}$. 
We find that every $\left.\frac{Q_{\mathbf{c}^{\prime}}}{q-1}\right|_{q=1}$ is zero unless $\mathbf{c}^{\prime} \in \mathbf{S}_{\mathbf{c}}$. Using the formula $\frac{\left|G l_{d_{i}}\left(\mathbb{F}_{q}\right)\right|}{\left|G l_{d_{i}-1}\left(\mathbb{F}_{q}\right)\right|}=$ $\left(q^{d_{i}}-1\right) q^{d_{i}-1}$ and L'Hospital's rule, we find $\left.\frac{Q_{\mathrm{c}^{i j}}}{q-1}\right|_{q=1}=d_{i} d_{j}$. By the decomposition $P_{\mathbf{c}}(q)=\frac{1}{q-1} \sum_{\mathbf{c}^{\min } \prec \mathbf{c}^{\prime} \preceq \mathbf{c}} Q_{\mathbf{c}^{\prime}}(q)$, we obtain the lemma.

4.2. Alternative proof. We now explain how the previous theorem gives a geometric version of the algebraic proof given in [BS] and in the present paper. First, as $\overline{\mathcal{O}_{\mathbf{c}}}$ is a cone, we claim that the rational smoothness of $\overline{\mathcal{O}_{\mathbf{c}}}$ implies that the cohomology of $X_{\mathbf{c}}$ is the same as the cohomology of the projective space, i.e. $\operatorname{dim} H^{i}\left(X_{\mathbf{c}}, \mathbb{Q}\right)=\operatorname{dim} H^{i}\left(\mathbb{P}^{d(\mathbf{c})-1}, \mathbb{Q}\right)$. Let's sketch a proof for this claim, following Bri98. By Remark 2.13 the rational smoothness property implies that $H^{i}\left(\overline{\mathcal{O}_{\mathbf{c}}}, \overline{\mathcal{O}_{\mathbf{c}}} \backslash\{0\}\right)=0, i \neq 2 d(\mathbf{c}), H^{2 d(\mathbf{c})}\left(\overline{\mathcal{O}_{\mathbf{c}}}, \overline{\mathcal{O}_{\mathbf{c}}} \backslash\{0\}\right)=\mathbb{Q}$. As $\overline{\mathcal{O}_{\mathbf{c}}}$ is contractible, we obtain by a long exact sequence in relative cohomology, that $\overline{\mathcal{O}_{\mathbf{c}}} \backslash\{0\}$ is a rational cohomology sphere of dimension $2 d(\mathbf{c})-1$. Now, let $S^{1} \subset \mathbb{C}^{*}$ acting naturally on the cone $\overline{\mathcal{O}_{\mathbf{c}}}$. It is clear that $X_{\mathbf{c}}$ and $\left(\overline{\mathcal{O}_{\mathbf{c}}} \backslash\{0\}\right) / S^{1}$ have the same rational cohomology. Hence, a Gysin exact sequence gives $H^{0}\left(X_{\mathbf{c}}\right) \simeq H^{2}\left(X_{\mathbf{c}}\right) \simeq \ldots H^{2(d(\mathbf{c})-1)}\left(X_{\mathbf{c}}\right) \simeq \mathbb{Q}$ and $H^{2 k+1}\left(X_{\mathbf{c}}\right)=0$. This proves the claim.

Now, $\chi\left(X_{\mathbf{c}}\right)=\chi\left(\mathbb{P}^{d(\mathbf{c})-1}\right)=d(\mathbf{c})$. By Theorem 4.1 this implies that $\operatorname{dim} \overline{O_{\mathbf{c}}}=$ $\operatorname{dim} E_{\mathbf{d}}(J(\mathbf{c}))$, and then $\overline{O_{\mathbf{c}}}=E_{\mathbf{d}}(J(\mathbf{c}))$. Once again, this implies that $\overline{O_{\mathbf{c}}}$ is smooth.

\section{ACKNOWLEDGEMENTS}

The first author wants to thank Michel Brion for suggesting the geometric approach and Markus Reineke for interesting and helpful email conversations.

The second author thanks Robert Bédard for several discussions on the subject.

\section{REFERENCES}

[BL00] S. Billey and V. Lakshmibai, Singular loci of schubert varieties, Progress in Math. 182, Birkhäuser Boston-Basel-Berlin, 2000.

[Bon95] K. Bongartz, Degenerations for representations of tame quivers, Annales Scientifiques De L'école Normale Supérieure (IV) 28 (1995), 647-668.

[BM] W.Borho and R.MacPherson. Partial resolutions of nilpotent varieties, in Analyse et topologie sur les espaces singuliers II, Astérisque 101-102, (1982), 23-74.

[BS] R. Bédard and R. Schiffler, Rational smoothness of varieties of representations for quivers of type A, preprint.

[Bri98] M. Brion, Equivariant cohomology and equivariant intersection theory, Representation Theory and Algebraic geometry, Kluwer Acad. Publ., (1998), 1-37.

[Cal] Ph. Caldero, A multiplicative property of quantum flag minors, Representation Theory 7, (2003), 164-176.

[Car94] J. Carrell, The Bruhat graph of a Coxeter group, a conjecture of Deodhar, and rational smoothness of Schubert varieties, p. 53-62 in Algebraic groups and their generalizations (W. Haboush and B. Parshall eds), American Mathematical Society, Providence 1994.

[Dan96] V.I. Danilov, Cohomology of algebraic varieties., ch. Algebraic geometry II, pp. 1-125, I.R. Shafarevich eds Encyclopedia Of Math. Sciences, 1996.

[Deo85] V. Deodhar, Local Poincaré duality and nonsingularities of Schubert varieties, Comm. Alg. 13, (1985), 1379-1388.

[Gre95] J.A. Green, Hall algebras, hereditary algebras and quantum groups, Invent.Math. 120 (1995), 361-377.

[Kas91] M. Kashiwara, On crystal bases of the q-analogue of the universal enveloping algebra, Duke Math. J. 63 (1991), $465-516$.

[Lus90a] G. Lusztig, Canonical bases arising from quantized enveloping algebras, J.Amer.Math.Soc. 3 (1990), 447-498.

[Lus90b] G. Lusztig, Finite dimensional Hopf algebras arising from quantized universal enveloping algebras, J.Amer.Math.Soc 3 (1990), 257-296. 
[Lus93] G. Lusztig, Introduction to quantum groups, Progress in Mathematics, 110, Birkäuser, (1993).

[Nör] R. Nörenberg, From elementary calculations to Hall polynomials, preprint.

[Rei99] M. Reineke, Multiplicative properties of dual canonical bases of quantum groups, Journal of Algebra 211 (1999), 134-149.

[Rie94] C. Riedtmann, Lie algebras generated by indecomposables, Journal of algebra, 170, (1994), 526-546.

[Rin90] C. M. Ringel, Hall algebras, vol. 26, pp. 433-447, Banach Center Publ., 1990.

[Rin93] C. M. Ringel, Hall algebras revisited, Israel Math.Conf.Proc. 7 (1993), 171-176.

Département de Mathématiques, Université Claude Bernard Lyon I, 69622 VilleurBanne Cedex, France

E-mail address: caldero@igd.univ-lyon1.fr

School of mathematics and statistics, Carleton University, 1125 Colonel By Drive, Room 4302 Herzberg Building, Ottawa, Ontario, Canada K1S 5B6

E-mail address: ralf@math.carleton.ca 